\title{
TRATAMENTO MEDICAMENTOSO DA OSTEOARTROSE DO JOELHO
}

\author{
DRUG THERAPY IN KNEE OSTEOARTHROSIS
}

\author{
Márcia Uchôa de Rezende', \\ Riccardo Gomes Gobbi²
}

\section{RESUMO}

O tratamento clínico da osteoartrite/artrose (OA) está sempre indicado e baseia-se no autocuidado feito pelo paciente e orientado pelo médico. $\mathrm{O}$ uso de medicamentos é complementar às medidas de emagrecimento, ganho de força, de propriocepção, de flexibilidade e de amplitude de movimento. Entre os medicamentos disponíveis para o tratamento da OA há os que são essencialmente analgésicos e que não interferem no curso da doença; bem como os anti-inflamatórios, controversos por seus efeitos colaterais e pelo seu papel na OA, porém, com propriedades analgésicas e anti-inflamatórias indiscutíveis; e, por fim, as drogas modificadoras de estrutura, que retardam a evolução da OA. As medicações ainda podem ser de uso tópico, intra-articular, oral e injetável (sistêmico). As várias apresentações de ácido hialurônico $(\mathrm{AH})$ mostram o poder analgésico da droga e há indícios de poder modificador de estrutura da cartilagem pela medicação. Há nível de evidência IA, para diacereína e para a glucosamina, de que retardam a evolução da OA. Mais tecnologia para diagnóstico e controle de tratamento da OA, bem como mais estudos multicêntricos são necessários para consolidar o poder do tratamento medicamentoso de outras drogas.

Descritores - Osteoartrose; Antraquinonas; Glucosamina; Condroitina; Ácido hialurônico

\section{ABSTRACT}

Clinical treatment for osteoarthritis (OA) is very important and is based on patient's self care and guided by the physician. Drug therapy is additional to losing weight, improving muscular strength, proprioception, flexibility and range of motion. Between the available drugs for osteoarthritis' treatment, some are basically analgesics and do not interfere on disease's progression; some are anti-inflammatory with good analgesic power but with side effects that compromise their prolonged usage; and the structure modifying drugs that slow down the progression of $O A$. The medications are presented in topic, oral, intra-muscular, intra-venous and intra-articular forms. The hyaluronic acid has various presentations with good analgesic effect and some evidence of structure modifying property. There is IA evidence level for the use of diacerhein and of glucosamine to slow down the disease. Still, more technology for diagnosis and therapy control of $O A$ is necessary to define the efficacy of other drugs.

Keywords - Osteoarthritis; Anthraquinone; Glucosamine; Condroitin; Hyaluronic acid

1 - Médica Assistente do Grupo de Joelho do Instituto de Ortopedia e Traumatologia - HC/FMUSP,

2 - Médico Preceptor do Instituto de Ortopedia e Traumatologia-HC/FMUSP.

Trabalho realizado no Instituto de Ortopedia e Traumatologia do HC-FMUSP.

Correspondência: Riccardo Gomes Gobbi, Rua João Moura, 1.381, apto. 124, Pinheiros, São Paulo,SP. E-mail: gobbi85@bol.com.br

Declaramos existência de conflitos de interesse com Aché, Genzyme, Merck do Brasil, Solvay, TRB Pharma, Zambon, Zodiac. Neste artigo, não favorecemos nenhum produto comercial.

Todas as informações são baseadas em literatura médica e não houve nenhuma forma de patrocínio para realização deste artigo pelas empresas responsáveis pelas medicações citadas. 


\section{INTRODUÇÃO}

Até algumas décadas atrás, o tratamento da osteoartrite ou artrose (OA) era limitado ao uso de analgésicos simples, anti-inflamatórios, medidas físicas (emagrecimento, reforço muscular e modalidades fisioterápicas), infiltrações com corticoides e, nos casos refratários e mais graves, o tratamento cirúrgico.

O entendimento progressivo da fisiopatologia da artrose no joelho, a percepção de que o processo não é puramente mecânico e/ou de envelhecimento, e o esclarecimento das vias inflamatórias envolvidas levaram à aplicação clínica de vários outros medicamentos.

Apesar de a cura da artrose ainda estar fora do alcance da medicina, já se discute a existência de drogas modificadoras da doença, que têm a capacidade de alterar a evolução da degeneração articular, retardando sua progressão, podendo até torná-la assintomática e, desse modo, evitando uma parcela dos procedimentos cirúrgicos de salvação. Como muitos esperam que, se a medicação é capaz de retardar a doença, também pode torná-la assintomática, boa parte dos estudos com as drogas modificadoras de estrutura são de curta duração e comparados com drogas de ação rápida para analgesia. A OA é uma doença de evolução lenta. Alterações radiográficas demoram três anos para ser observadas/mensuradas. A própria perda do espaço articular do joelho está mais relacionada com a extrusão do menisco do que com a perda de cartilagem fêmoro-tibial.

Ao longo dos anos, muitos aprenderam que se pode controlar a dor na OA sem se intervir no processo da doença. E o oposto também ocorre. Pode-se intervir no processo da OA sem se resolver toda a dor. A dor é multifatorial e também relacionada com o grau de OA.

Marcadores de OA (no sangue e na urina) estão sendo pesquisados, exatamente para controlar se a artrose está em evolução ou sob controle.

Nesta atualização, abordaremos basicamente os medicamentos que são considerados modificadores de estrutura na doença e qual o nível de evidência que respalde seus usos.

\section{FISIOPATOLOGIA DA OA}

Apesar de OA ser uma doença de toda a articulação (cartilagem, ligamentos, sinóvia e osso), a lesão inicial costuma ser na cartilagem articular. A OA tem um forte componente genético e, na maioria das vezes, tem a sobrecarga mecânica como um iniciador do processo de lesão da cartilagem, que acaba evoluindo para um ciclo vicioso inflamatório, perpetuando a degradação articular. Essa via inflamatória tem como agentes primários a interleucina-1 (IL-1) e o fator de necrose tumoral (TNF), que induzem maior expressão de metaloproteases e óxido nítrico (NO), os principais agentes catabólicos produzidos pelos condrócitos em resposta à lesão, além de mais IL-1. O tratamento da artrose pode ter como alvo tanto a sobrecarga mecânica que leva à lesão articular quanto o ciclo inflamatório que perpetua essa lesão, em um ou vários pontos dessa cascata.

Para facilitar o estudo das opções medicamentosas utilizadas na artrose, costuma-se dividir as drogas em dois grandes grupos: o das drogas sintomáticas de ação rápida e o de ação lenta; dentre as últimas, estão as drogas modificadoras de estrutura (que também incluem drogas analgésicas de ação rápida e prolongada), que serão apresentadas a seguir. Deve ser sempre lembrado que a terapia farmacológica tem que ser vista como um complemento, nunca uma substituição, à terapia não medicamentosa. $\mathrm{O}$ objetivo primário do tratamento conservador da OA é o autocuidado (emagrecimento e controle motor $)^{(1)}$.

\section{DROGAS ESPECÍFICAS PARA 0 TRATAMENTO DA OA}

Dentro deste grupo estão as medicações que agem mais especificamente no processo patológico da artrose, tendo em teoria a capacidade de ser modificadoras da doença (prevenindo, retardando, revertendo ou estabilizando a alteração da estrutura articular). Existem evidências que sugerem que algumas dessas drogas sejam modificadoras da estrutura.

\section{ÁCIDO HIALURÔNICO}

O líquido sinovial na osteoartrose (OA) apresenta redução na sua viscoelasticidade ${ }^{(2,3)}$. Para a lubrificação e proteção das células e tecidos articulares, alta viscoelasticidade é fundamental. Desse modo, uma das causas da dor e diminuição da mobilidade articular pode ser a diminuição do efeito protetor desse meio viscoelástico nos receptores dolorosos do tecido sinovial ${ }^{(4)}$. A viscossuplementação é uma terapêutica relativamente nova, que age diretamente numa das causas da dor e rigidez na OA, substituindo o meio sinovial de baixa viscoelasticidade por uma solução de ácido hialurônico de alta viscoelasticidade $^{(5-7)}$. 
Existem no mercado vários preparados de ácido hialurônico e as suas diferentes propriedades reológicas são dependentes basicamente de seus pesos moleculares $^{(8)}$. Com um peso molecular de $6 \times 10^{6}$ daltons $(\mathrm{Da})$, as propriedades viscoelásticas do Hylan G-F 20 são similares às do líquido sinovial de adultos jovens saudáveis ${ }^{(3)}$; três injeções semanais são comprovadamente seguras e superiores em eficácia em relação ao placebo (infiltração com solução salina ou artrocentese) $)^{(9,10)}$ com analgesia de até seis meses. Com peso molecular de $500-730 \mathrm{kDa}$, as propriedades viscoelásticas do hialuronato de sódio (Na-HA) são menores do que as do líquido sinovial normal, porém se mostraram viscoindutores (isto é , induzem a produção de ácido hialurônico de maior peso molecular pela sinóvia) ${ }^{(11)}$. Os resultados desses preparados com menor peso molecular em comparação com injeção de solução salina ou artrocentese são variáveis, ora com diferença estatística, ora sem diferença $\mathrm{a}^{(12-14)}$.

Estudos comparando o preparado de alto peso molecular (Hylan G-F 20) com outros de baixo peso foram publicados nos últimos anos, com resultados variáveis. Alguns não mostraram diferença estatística ${ }^{(15)}$, enquanto outros, maior eficácia do composto com alto peso molecular ${ }^{(16-18)}$ e outros ainda, maior eficácia do composto de baixo peso molecular ${ }^{(11,19,20)}$. Outro estudo ainda mostrou similaridade entre o tratamento com ácido hialurônico e o tratamento apenas com medidas físicas/fisioterapia ${ }^{(18)}$.

Uma meta-análise recente ${ }^{(21)}$ mostrou que o uso do ácido hialurônico tem efeito pequeno quando comparado com o do placebo intra-articular e que os compostos com alto peso molecular tendem a apresentar melhores resultados (de analgesia rápida e prolongada na $\mathrm{OA}$ ).

$\mathrm{O}$ ácido hialurônico pode ter efeito modificador de estrutura. Um estudo randomizado ${ }^{(22)}$, em que os pacientes eram submetidos à artroscopia do joelho no início e no final do tratamento de um ano com quatro séries de três infiltrações semanais de ácido hialurônico $\left(6-8 \times 10^{5}\right)$, mostrou menos deterioração da cartilagem e mais organelas de síntese na cartilagem tratada com ácido hialurônico dos que na cartilagem injetada com solução salina. Um estudo prospectivo, randomizado e $\operatorname{cego}^{(23)}$, não mostrou diferença radiológica entre o grupo placebo e o da medicação em um ano, apesar de o tempo ser muito curto para avaliar diferenças radiológicas num método de tratamento de artrose; porém, foi observado que, separando apenas o grupo de pacientes com maior espaço articular (artrose menos severa, onde há mais condrócitos para receberem a influência, ou não, do medicamento), o ácido hialurônico mostrou resultado significativamente melhor.

Complicações possíveis são infecção (risco similar ao da injeção de corticoide) e reação inflamatória local que ocorre em até $3 \%$ dos casos, com sintomas durando até três semanas.

Desse modo, existem evidências do bom e prolongado efeito analgésico do ácido hialurônico, e de que, quanto maior seu peso molecular, maior seu poder analgésico. Qual o peso molecular que melhor preservaria a cartilagem articular, não se sabe ao certo. Alguns estudos em animais tendem a sugerir que o peso molecular entre 0,6 e $1,0 \times 10^{6} \mathrm{Da}$ seria melhor estimulador da produção de componentes da matriz, o que poderia ser parcialmente explicado porque o peso molecular menor penetraria a matriz extracelular mais facilmente, maximizando sua concentração e, também, facilitando sua interação com as células-alvo da sinóvia. Além disso, há evidências de que a ligação das moléculas de ácido hialurônico com os receptores celulares é dependente do peso molecular ${ }^{(11)}$. Há estudos que mostram que o $\mathrm{AH}$ de alto peso molecular também estimula as organelas de síntese ${ }^{(24)}$. Essa observação de qual peso molecular protege melhor a cartilagem articular em animais ainda não se comprovou em humanos ${ }^{(11)}$.

Há preparados que são extraídos da crista do galo; então, pacientes alérgicos a produtos de ave não devem ser injetados com tais preparações. Há ácido hialurônico produzido por fermentação, que tem menor peso molecular, porém sem os problemas de alérgenos ou de ligações cruzadas entre moléculas de ácido hialurônico que podem levar a sinovite durante o processo de quebra dessas ligações cruzadas.

O ácido hialurônico não deve ser injetado em joelhos com sinovite. A sinovite dever ser tratada primeiramente, ou puncionada e junto com a primeira ampola de ácido hialurônico, pode-se injetar $1 \mathrm{ml}$ de triancinolona ${ }^{(25)}$.

\section{DIACEREÍNA}

Atua principalmente pela inibição dos efeitos da IL-1 esta degrada a proteína inibidora do fator nuclear capa beta, que leva à transcrição de óxido nítrico, IL-1, TGF e metaloproteinases ${ }^{(26-29)}$ e também tem propriedades anabólicas, estimulando a produção de TGF-B e proteoglicanos, colágeno e ácido hialurônico ${ }^{(30)}$. 
Além de eficaz no alívio sintomático da artrose, foi comprovada em estudo de longo prazo (três anos), multicêntrico e prospectivo, a ação modificadora da doença pela menor diminuição do espaço articular em relação ao placebo, isto é, nível de evidência IA de que é uma droga modificadora da doença osteoartrítica, retardando a evolução da $\mathrm{OA}^{(31)}$.

A dosagem recomendada é de $100 \mathrm{mg} / \mathrm{dia}^{(32)}$; atenção deve ser dada ao possível efeito colateral de alterar o peristaltismo intestinal. Pode ser administrada a pacientes com insuficiência renal e cardíaca ${ }^{(31,32)}$.

\section{GLUCOSAMINA}

A glucosamina participa da síntese das glicosaminoglicanas (GAGs), proteoglicanas e hialuronato da cartilagem articular, apesar de o mecanismo exato ainda não estar elucidado ${ }^{(33)}$. Além de funcionar como substrato, age diretamente no condrócito, estimulando a síntese de proteoglicanas e inibindo a de metaloproteases. Inibe os efeitos da IL-1 sobre o fator nuclear capa beta dentro do condrócito, também inibindo a produção de óxido nítrico, mais IL-1 e TGF. Tem ação sobre o osteoclasto e sobre a sinóvia ${ }^{(34)}$.

Meta-análises recentes mostraram que a droga tem eficácia superior à do placebo, tendo, além da melhora sintomática, um efeito de diminuir o estreitamento articular ${ }^{(33-36)}$. Há três tipos de glucosamina no mercado. A glucosamina hidroclorídrica $(\mathrm{HCl}$ - retirada da casca de caranguejo), a glucosamina sulfatada (retirada da casca de camarões de águas profundas) e a glucosamina sintética (sulfatada). Há nível de evidência IA de que a glucosamina sintética retarda a evolução da OA (uso contínuo por três anos) ${ }^{(37)}$ e esse efeito se mantém mesmo após a suspensão do uso da droga por cinco $\operatorname{anos}^{(38)}$. Sua posologia é simples. Dose de $1.500 \mathrm{mg}$ diários. No caso da glucosamina sintética, essa dose leva a uma concentração de $10 \mu \mathrm{Mol}$ no sangue e líquido sinovial (mínima dose terapêutica da glucosamina). A glucosamina hidroclorídrica, dada na dose de $500 \mathrm{mg}$ três vezes ao dia, chega em concentração de $3 \mu \mathrm{Mol}$ no líquido sinovial (menor do que a da dose

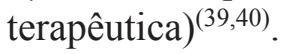

\section{CONDROITINA}

O sulfato de condroitina é uma GAG encontrada em vários tecidos humanos, inclusive na cartilagem hialina. Estudos mostram, além de estimulação direta da cartilagem, ação de inibição da IL-1 e metaloproteases ${ }^{(41,42)}$.
A condroitina é uma molécula grande, que é quebrada ao ser absorvida pelo intestino. Na sua formação, há glucosamina.

Meta-análises recentes mostraram que a droga tem eficácia superior à do placebo, com efeito predominante no alívio dos sintomas, sem diminuir o estreitamento articular da glucosamina ${ }^{(33,35,36,43)}$.

A dose recomendada é a de $1.200 \mathrm{mg} / \mathrm{dia}$.

\section{ASSOCIAÇÃO DE GLUCOSAMINA E CONDROITINA}

A associação das drogas, por agirem em vias diferentes tendo efeitos complementares, seria melhor do que o uso isolado. A dosagem usual é de $1.500 \mathrm{mg}$ de glucosamina e $1.200 \mathrm{mg}$ de condroitina em dose única diária ou dividida, dependendo da apresentação comercial. Apresenta boa tolerabilidade ao uso prolongado, com poucos efeitos colaterais.

$\mathrm{O}$ estudo GAIT (Glucosamine HCl/chondroitin Arthritis Intervention Trial), multicêntrico, duplo-cego, randomizado, controlado com grupo placebo e grupo celecoxibe (AINH inibidor seletivo da COX-2) foi publicado recentemente ${ }^{(44)}$. Foram incluídos 1.583 pacientes e os resultados após 24 semanas mostraram que no grupo geral a associação das drogas, do ponto de vista de analgesia, não foi superior à do placebo, enquanto o celecoxibe o foi. Separando-se apenas os pacientes com dor moderada ou severa, houve diferença estatística em relação ao grupo placebo, sugerindo maior benefício nesse grupo de pacientes.

\section{INSAPONIFICÁVEIS DO ABACATE E SOJA}

São comprovadamente inibidores de IL-1, IL-6, IL-8 e metaloproteases in vitro ${ }^{(45)}$ e estimuladores do condrócito in vitro ${ }^{(46)}$.

Um artigo de revisão de quatro estudos clínicos randomizados, duplo-cegos e controlados por grupo placebo mostrou melhora dos sintomas em três deles, porém, o único desses estudos que era de longa duração mostrou resultados negativos para artrose de quadril, mas positivos para OA de joelho ${ }^{(47)}$. Uma meta-análise recente (2008) de estudos controlados com insaponificáveis de soja e de abacate mostrou que seu uso reduz a dor, reduz a ingesta de analgésicos e melhora a função de pacientes com OA de joelhos ${ }^{(48)}$. Apesar disso, ainda 
não está esclarecida a capacidade dessa droga de prevenir a perda do espaço articular na artrose. Um estudo prospectivo randomizado avaliando o espaço articular do quadril após uso da medicação mostrou resultado significativo apenas no subgrupo com artrose avançada, não sendo significativo na população estudada como um todo ${ }^{(49)}$.

Com dosagem de $300 \mathrm{mg} / \mathrm{dia}$, o consenso brasileiro de OA (OARSI) indica o uso de insaponificáveis de soja e de abacate para o tratamento da OA.

\section{CLOROQUINA}

Apresenta efeito de supressão da produção de NO induzida por IL-1 ${ }^{(50)}$.

Sua eficácia clínica no tratamento da artrose não é estabelecida, porém, parece ser útil principalmente nas formas inflamatórias e erosivas da artrose. No Brasil, há consenso de indicação (OARSI) da cloroquina como forma de tratamento da OA.

\section{REFERÊNCIAS}

1. Recommendations for the medical management of osteoarthritis of the hip and knee: 2000 update. American College of Rheumatology Subcommittee on Osteoarthritis Guidelines. Arthritis Rheum. 2000;43(9):1905-15.

2. Balazs EA. Viscoelastic properties of hyaluronic acid and biological lubrication. Univ Mich Med Cent J. 1968;Suppl:255-59.

3. Balazs EA. The physical properties of synovial fluid and the special role of hyaluronic acid. In: Helfet A, ed. Disorders of the knee. 2nd ed. Philadelphia: J.B. Lippincott; 1982. p. 61-74.

4. Pozo MA, Balazs EA, Belmonte C. Reduction of sensory responses to passive movements of inflamed knee joints by hylan, a hyaluronan derivative. Exp Brain Res. 1997;116:3-9.

5. Balazs EA, Denlinger JL. Clinical uses of hyaluronan. In: Evered D, Whelan J, eds. The Biology of Hyaluronan (Ciba Foundation Symposium \#143). Chichester, England: John Wiley \& Sons; 1989. p. 265-80.

6. Balazs EA, Denlinger JL. Viscosupplementation: A new concept in the treatment of osteoarthritis. J Rheumatol. 1993;20(Suppl 39):3-9.

7. Balazs EA, Denlinger JL. The role of hyaluronic acid in arthritis and its therapeutic use. In: Peyron JG, editor. Osteoarthritis-current clinical and fundamental problems. Rueil-Malmaison: Laboratories CibaGeigy; 1985. p. 165-74.

8. Adams ME, Lussier AJ, Peyron JG. A risk-benefit assessment of injections of hyaluronan and its derivatives in the treatment of osteoarthritis of the knee. Drug Saf. 2000;23(2):115-30.

9. Wobig M, Dickhut A, Maier R, Vetter G. Viscosupplementation with hylan G-F20: a 26 week controlled trial of efficacy and safety in the osteoarthritis knee. Clin Ther. 1998;20(3):410-23.

10. Scale D, Wobig M, Wolpert W. Viscosupplementation of osteoarthritic knees with hylan: a treatment schedule study. Curr Ther Res. 1994:55:220-32.

\section{OUTRAS DROGAS}

Muitas outras substâncias, de origem natural ou não, vêm sendo estudadas por um suposto efeito antiartrósico. Até mesmo a terapia gênica vem sendo investigada para aplicação no tratamento da artrose.

Devemos esperar muito ainda do futuro no tratamento clínico da osteoartrose.

\section{CONSIDERAÇÕES FINAIS}

Como visto, existem várias medicações disponíveis com o potencial de modificar a progressão da artrose por agirem diretamente sobre a fisiopatologia da doença. Apesar disso, com base nas evidências científicas, pode-se concluir que:

- O ácido hialurônico melhora a dor e a função do paciente com artrose;

- A diacereína e a glucosamina sintética retardam a progressão da artrose.

11. Ghosh P, Guidolin D. Potencial mechanism of action of intra-articular hyaluronan therapy in osteoarthritis: are the effects molecular weight dependent? Semin Arthritis Rheum. 2002;32(1):10-37.

12. Lohmander LS. A controlled, randomized, double-blind multicenter trial of intra-articular hyaluronan treatment in osteoarthrosis of the knee. Acta Orthop Scand. 1995;66(Suppl 265):35-6.

13. Dahlberg L, Lohmander LS, Ryd L. Intraarticular injections of hyaluronan in patients with cartilage abnormalities and knee pain. A one-year double-blind, placebo controlled study. Arthritis Rheum. 1994;37(4):521-8.

14. Altman RD, Moskowitz R, for the Hyalgan Study Group. Intraarticular sodium hyaluronate $\left(\mathrm{Hyalgan}^{\circledR}\right)$ in the treatment of patients with osteoarthritis of the knee: a randomized clinical trial. J Rheumatol. 1998;25(11):2203-12.

15. Karlsson J, Sjögren LS, Lohmander LS. Comparison of two hyaluronan drugs and placebo in patients with knee osteoarthritis. A controlled, randomized, double-blind, parallel-design multicentre study. Rheumatology. 2002;41(11):1240-8.

16. Wobig M, Bach G, Beks P, Dickhut A, Runzheimer J, Schwieger G, et al. The role of elastoviscosity in the efficacy of viscosupplementation for osteoarthritis of the knee: a comparison of hylan G-F 20 and a lower-molecular-weight hyaluronan. Clin Ther. 1999;21(9):1549-62.

17. Goorman SD, Watanabe TK, Miller EH, Perry C. Functional outcome in the knee osteoarthritis after treatment with hylan G-F20: a prospective study. Arch Phys Med Rehabil. 2000;81(4):479-83.

18. Atamaz F, Kirazli Y, Akkoc Y. A comparison of two different intraarticular hyaluronan drugs and physical therapy in the management of knee osteoarthritis. Rheumatol Int. 2006;26(10):873-8

19. AsariA, Miyauchi S, Matsuzaka S, Ito T, Kominami E, Uchiyama Y. Molecular weight-dependent effects of hialuronate on the arthritic synovium. Arch Histol Cytol. 1998;61(2):125-35. 
20. ShimizuC, Kubo T, Hirasawa Y, Coutts RD, Amiel D. Histomorphometric and biochemical effect of various hyaluronans on early osteoarthritis. J Rheumatol. 1998;25(9):1813-9.

21. Lo GH, La Valley M, McAlindon T, Felson DT. Intraarticular hyaluronic acid in treatment of knee osteoarthritis: a metaanalysis. JAMA. 2003;290(23):315-21.

22. ListratV, Ayral X, Patarnello F, Bonvarlet JP, Simonnet J, Amor B, et al. Arthroscopic evaluation of potential structure modifying activity of hyaluronan (Hyalgan) in osteoarthritis of the knee. Osteoarthritis Cartilage. 1997;5(3):153-60.

23. Jubb RW, Piva S, Beinat L. Structure modifying study of hyaluronan (500-700kDa, Hyalgan) in osteoarthritis of the knee. Arthritis Rheum. 2001;44(Suppl):S155.

24. GoldbergVM, Buckwalter JA. Hyaluronans in the treatment of osteoarthritis of the knee: evidence for disease-modifying activity. Ostearthritis Cartilage. 2005;13(3):216-24.

25. Ozturk C, Atamaz F, Hepguler S, Argin M, Arkum R. The safety and efficacy of intraarticular hyaluronan with/without corticosteroid in knee ostearthritis: 1-year, single-blind, randomized study. Rheumatol Int. 2006;26(4):314-9.

26. Mendes AF, Caramona MM, Carvalho AP, Lopes MC. Diacerhein and rhein prevent interleukin-1beta-induced nuclear factor-kappaB activation by inhibiting the degradation of inhibitor kappaB-alpha. Pharmacol Toxicol. 2002;91(1):22-8.

27. Martin G, Bogdanowicz P, Domagala F, Ficheux H, Pujol JP. Rhein inhibits interleukin-1 beta-induced activation of MEK/ERK pathway and DNA binding of NF-kappa B and AP-1 in chondrocytes cultured in hypoxia: a potential mechanism for its disease-modifying effect in osteoarthritis. Inflammation. 2003;27(4):233-45.

28. Martel-Pelletier J, Mineau F, Jolicoeur FC, Cloutier JM, Pelletier JP. In vitro effects of diacerein and rhein on IL-1 and TNF-a systems in human osteoarthritic synovium and chondrocytes. J Rheumatol. 1998;25(4):753-62.

29. Moore AR, Greenslade KJ, Alam CA, Willoughby DA. Effects of diacerhein on granuloma induced cartilage breakdown in the mouse. Osteoarthritis Cartilage. 1998;6(1):19-23.

30. Pujol JP. Collagenolytic enzymes and interleukin-1: their role in inflammation and cartilage degradation. The antagonistic effects of diacerhein on IL-1 actions on cartilage matrix components. Laboratoire de Biochimie du Tissu Conjonctif, CHU Côte de Nacre, Caen, France. 1991.

31. Dougados M, Nguyen M, Berdah L, Maziéres B, Vignon E, Lequesne M; ECHODIAH Investigators Study Group. Evaluation of the structure-modifying effects of diacerein in hip osteoarthritis: ECHODIAH, a three year, placebo-controlled trial: evaluation of the chondromodulating effect of diacerein in OA of the hip. Arthritis Rheum. 2001;44(11):2539-47.

32. PelletierJP, Yaron M, Haraoui B, Cohen P, Nahir MA, Choquette D, et al. Efficacy and safety of diacerein in osteoarthritis of the knee: a double-blind, placebo-controlled trial. The Diacerein Study Group. Arthritis Rheum. 2000;43(10):2339-48.

33. Towheed TE, Maxwell L, Anastassiades TP, Shea B, Houpt J, Robinson V, et al. Glucosamine therapy for treating osteoarthritis. Cochrane Database Syst Rev. 2005;(2):CD002946.

34. Jimenez S, Dodge G. The effects of glucosamine sulfate on human chondrocyte gene expression. Osteoarthritis Cartilage. 1997;5(SA):72.
35. RichyF, Bruyere O, Ethgen O, Cucherat M, Henrotin Y, Reginster JY. Structural and symptomatic efficacy of glucosamine and chondroitin in knee osteoarthritis: a comprehensive meta-analysis. Arch Intern Med. 2003;163(13):1514-22.

36. McAlindon TE, LaValley MP, Gulin JP, Felson DT. Glucosamine and chondroitin for treatment of osteoarthritis: a systematic quality assessment and meta-analysis. JAMA. 2000;283(11):1469-75.

37. ReginsterJY, Deroisy R, Rovati LC, Lee RL, Lejeune E, Bruyere O, et al. Long-term effects of glucosamine sulphate on osteoarthritis progression: a randomised, placebo-controlled clinical trial. Lancet. 2001;357(9252):251-6.

38. Bruyere O, Pavelka K, Rovati LC, Gatterova J, Gaicovelli G, Olejarova $M$, et al. Total joint replacement after glucosamine sulphate treatment in knee osteoarthritis: results of a mean 8-year observation of patients from two previous 3 -year, randomized, placebo-controlled trials. Ostearthritis Cartilage. 2008;16(2):254-60.

39. Persiani S, Roda E, Rovati LC, Locatelli M, Giacovelli G, Roda A. Oral bioavailability and plasma pharmacokinetics after increasing doses of crystalline glucosamine sulfate in man. Osteoarthritis Cartilage. 2005;13(12):1041-9.

40. Persiani S, Rotini R, Trisolino G, Rovati LC, Locatelli M, Paganini D, et al. Synovial and plasma glucosamine concentrations in osteoarthritic patients following oral crystalline glucosamine sulphate at therapeutic dose. Osteoarthritis Cartilage. 2007;15(7):764-72.

41. Karzel K, Lee KJ. Effect of hexosamine derivatives on mesenchymal metabolic processes of in vitro culture fetal bone explants. J Z Rheumatol. 1982;41(5):212-8.

42. Mathieu P. A new mechanism of action of chondroitin sulfates ACS4ACS6 in osteoarthritic cartilage. Presse Med. 2002;31(29):1383-5.

43. Leeb RF, Schweitzer $H$, Montag K, Smolen JS. A metaanalysis of chondroitin sulfate in the treatment of osteoarthritis. J Rheumatol. 2000;27(1):205-11.

44. CleggDO, Reda DJ, Harris CL, Klein MA, O'Dell JR, Hooper MM, et al. Glucosamine, chondroitin sulfate, and the two in combination for painful knee osteoarthritis. N Engl J Med. 2006;354(8):795-808.

45. Henrotin YE, Sanchez C, Deberg MA, Piccardi N, Guillou GB, Msika P, et al. Avocado/soybean unsaponifiables increase aggrecan synthesis and reduce catabolic and proinflammatory mediator production by human osteoarthritic condrocytes. J Rheumatol. 2003;30(8):1825-34.

46. MauvielA, Daireaux M, Hartmann DJ, Galera P, Loyau G, Pujol JP. Effects of unsaponifiable extracts of avocado/soybeans (PIAS) on the production of collagen by cultures of synoviocytes, articular chondrocytes and skin fibroblasts. Rev Rhum Mal Osteoartic. 1989;56(2):207-11.

47. Ernst E. Avocado/soybean unsaponifiables (ASU) for osteoarthritis: a systematic review. Clin Rheumatol. 2003;22(4-5):285-8.

48. Christensen R, Bartels EM, Astrup A, Bliddal H. Symptomatic efficacy of avocado-soybean unsaponifiables (ASU) in ostearthritis (OA) patients: a meta-analysis of randomized controlled trials. Ostearthritis Cartilage. 2008;16(4):399-408

49. Lequesne M, Maheu E, Cadet C, Dreiser RL. Structural effect of avocado/soybean unsaponificables on joint space loss in osteoarthritis of the hip. Arthritis Rheum. 2002;47(1):50-8.

50. Vuolteenaho K, Kujala P, Moilanen T, Moilanen E. Aurothiomalate and hydroxychloroquine inhibit nitric oxide production in chondrocytes and in human osteoarthritic cartilage. Scand J Rheumatol. 2005;34(6):475-9. 